\title{
RESEARCH OF FERROMAGNETIC NANOPARTICLES TRANSPORT UNDER MAGNETIC FIELD
}

\author{
Maxim Pryazhnikov ${ }^{1,}$, Dmitriy Guzey ${ }^{1}$, Andrey Minakov ${ }^{1,2}$, Vladimir Zhigarev $^{1}$, and Anna \\ Shebeleva ${ }^{1}$ \\ ${ }^{1}$ Siberian Federal University \\ ${ }^{2}$ Institute of Thermophysics SB RAS
}

\begin{abstract}
During the research we conducted an experimental study and numerical simulation of ferromagnetic nanoparticles transport behavior in a constant magnetic field. The growth dynamics of nanoparticle deposits on channel walls was studied depending on the Reynolds number and the intensity of the magnetic field. We obtained flow pattern, the concentration field and the trajectory of nanoparticles depending on the Reynolds number. The simulation results were compared with data from experiments. There was good qualitative and quantitative agreement between calculation and experiment.
\end{abstract}

\section{Experimental study}

During the research we conducted the experimental study of the behavior of ferromagnetic nanoparticles flowing in a liquid in a constant magnetic field. According to the study an experimental setup was a cylindrical tube, made of glass with an internal diameter of $6 \mathrm{~mm}$ and a length of $50 \mathrm{~cm}$. The tube was connected to a pump and a container with liquid via hoses. Below a permanent neodymium magnet of 50x30x10 mm was brought to the glass tube. Distilled water with the nanoparticles was pumped through the tube. The particles interacted with the magnetic field, and deposited on the tube wall. For pumping liquid we used gear pump WT30001JA. Pump drives allowed to pump various fluids at a rate of 85.7 to $2571.4 \mathrm{ml} / \mathrm{min}$.

Investigation of particle deposition process was conducted with a ferromagnetic fluid based on distilled water with the nanoparticles $\mathrm{Fe}_{3} \mathrm{O}_{4}$. Nanopowders had been acquired in JSC "Plazmoterm" (Moscow). Volumetric concentration of particles was very low, $10^{-3} \%$. The size of the nanoparticles was $50 \mathrm{~nm}$. In order to prepare the ferrofluid we used a standard two-step method. The required amount of the powder was added to the liquid, after which the resulting suspension was thoroughly stirred. To destroy nanoparticle conglomerates the suspension was treated in the ultrasound bath "Sapphire" SC-10338 during 45 minutes.

\footnotetext{
* Corresponding author: arrivent@yandex.ru
} 
We considered the permanent magnetic field produced by the neodymium magnet, the

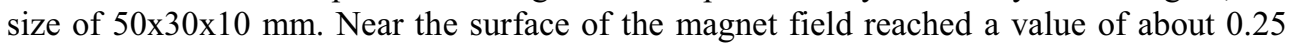
Tesla. The field was measured with the help of a milliteslameter TPU. A high-speed camera was used to fix the deposition of particles in the tube in a constant magnetic field.

The flow rate and the magnetic field strength varied during the experiments. Two series of measurements were conducted. The first series was held to study the effect of nanofluid flow on shape of particles deposition on the tube. The magnet was located under the tube, $\mathrm{ym}=0 \mathrm{~mm}$. Five mass flows were considered. Mass flow correspond to the following Reynolds numbers: $1200,2810,4220,5760,7145$. The results of the study are presented in the form of sequential frames for the flow of liquid when the number of $R e=2810$ (every 10 seconds) in figure 1.

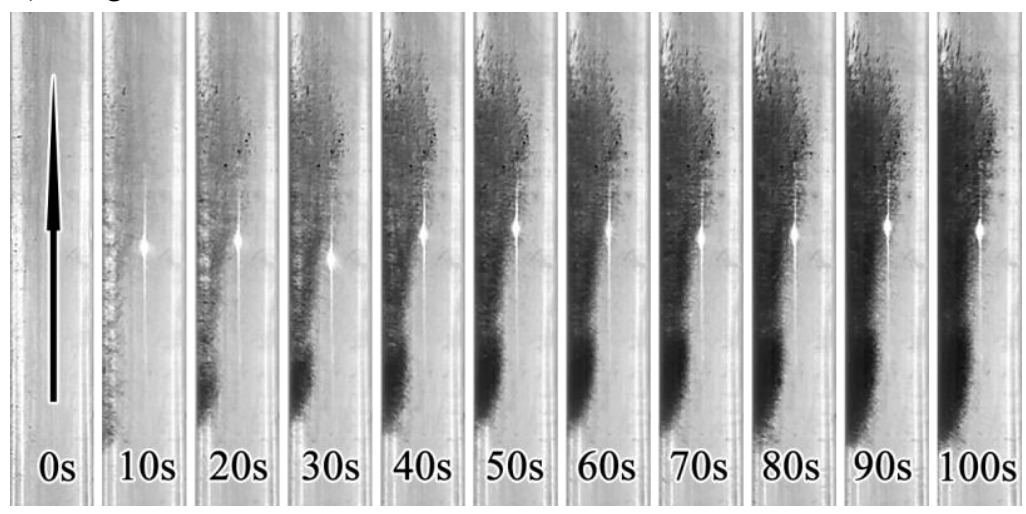

Fig. 1. The deposition of particles in a constant magnetic field at $\operatorname{Re}=2810$.

We obtained a dynamics of the formation of particles deposition at different Reynolds numbers. In addition, we made a comparison of deposition shapes of Reynolds numbers 60 seconds after the start of the nanoparticles deposition process in the tube. With the increase of the Reynolds number the growth rate of deposition and the height itself nanoparticle deposition height were being reduced. It was shown that with increasing Reynolds number, length of the deposition zone in the tube is reduced.

The second series was conducted in order to investigate the influence of the magnetic field intensity on particle formation deposition during the time. Liquid was pumped at a constant rate, the value of which corresponded to the Reynolds number $R e=1200$. Three variants of magnet position were considered: below a tube onto $y_{m}=0$, below tube onto $y_{m}$ $=14 \mathrm{~mm}$, and onto $y_{m}=28 \mathrm{~mm}$. As a result of experimental studies it was found that with the weakening of the magnetic field the deposition height is reduced. The height of the particles increased during deposition time. About a minute after the start of the experiment the growth rate reached a maximum. Then a dynamic equilibrium between deposition and entrainment of the particles was achieved, and deposits form was stabilized. It is also obvious that the position of the magnet (magnetic field) influences the shape of the particles deposition: the smaller the magnetic field is, the greater the flow of entrained particles is and the less deposits are on the tube.

\section{Numerical simulation}

Numerical simulation of transport of nanoparticles in a constant magnetic field was conducted.To simulate the transport of nanoparticles in the magnetic field we used a novel Euler-Lagrange combined two-component approach. As part of the Lagrange approach for the base fluid the equations of continuity, momentum and energy were being solved, and 
the movement of the particles was modeled on the basis of Newton's second law by solving an ODE. Euler two-component model describes nanoparticles in a liquid as a binary mixture, one of which components is nanoparticles. Statement of the problem completely repeated the experimental study. The computational domain is a circular channel with an inner diameter of $6 \mathrm{~mm}$ and a length of $50 \mathrm{~cm}$. A computational grid consisting of 500 thousand nodes, was used for the simulation.. fig. 2-3 shows the results of calculations (vector velocity field in the channel, the trajectory of the particles) at $\operatorname{Re}=2810$.

By increasing the Reynolds number the flow was being complicated and the shape of the vortex field in the channel wall was being changed. The deposits form was also changed. Nevertheless, the calculation perfectly reproduces the shape of deposition observed in the experiment (see fig. 4). With a flow rate of increase in the action of magnetic force compared to inertial forces are weakened.

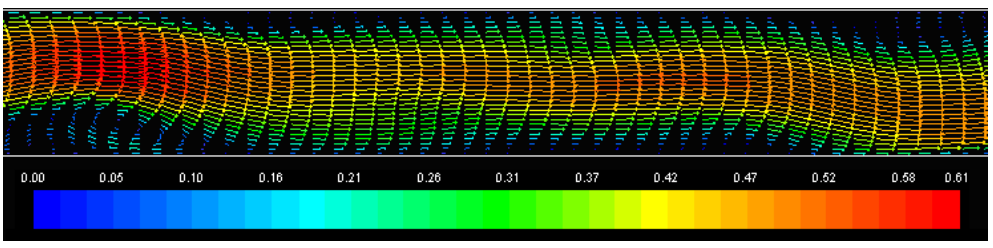

Fig. 2. The vector velocity field in the channel at $\mathrm{Re}=2810$.

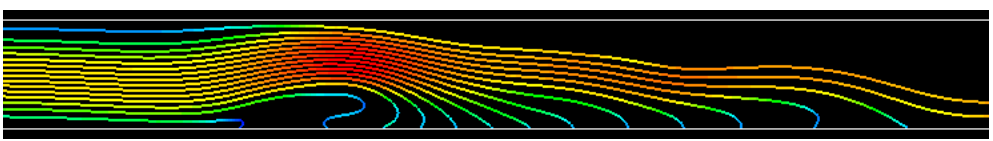

Fig. 3. Trajectories of nanoparticles at $\operatorname{Re}=2810$.

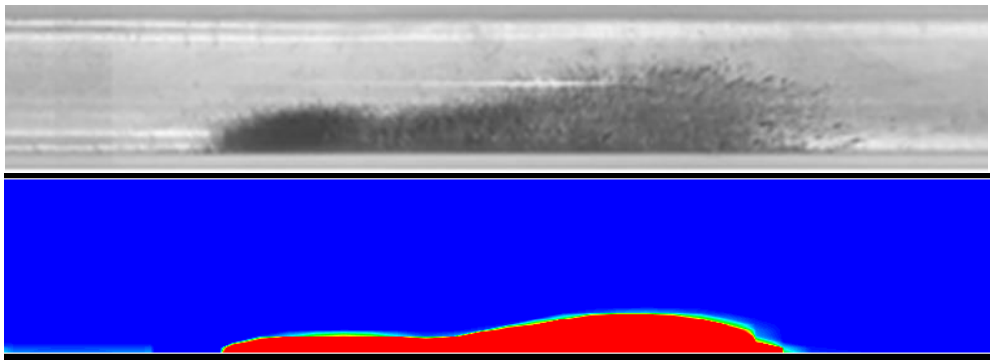

Fig. 4. Comparison of shape of particle deposition in the channel at $R e=2810$ (top - experiment, bottom - numerical simulation).

\section{Conclusions}

1. In this research we conducted the experimental study of nanoparticle transport in a constant magnetic field. During our experiments we studied the dynamics of nanoparticles depositions growth on the channel walls depending on the Reynolds number and the magnetic field strength. For the first time we obtained characteristic profiles of nanoparticle deposition zones, determined the dimensions of the deposition rate of their formation depending on the Reynolds numbers and the magnetic field strength.

2. We conducted a numerical simulation of transport of nanoparticles in a constant magnetic field. To simulate the transport of nanoparticles in the magnetic field we used a novel Euler-Lagrange combined two-component approach.

3 . We conducted the verification and adaptation of mathematical models of magnetic nanoparticles transport under the influence of external electromagnetic fields on the data of 
the experiment. We obtained the flow pattern, the concentration field and the trajectory of nanoparticles as a function of the Reynolds number. The simulation results were compared with experimental data, there was good qualitative and quantitative agreement between calculation and experiment.

\section{Acknowledgments}

This work was financially supported by the Ministry of Education and Science of Russia (Contract No. 14.607.21.0104 (RFMEFI60714X0104)).

\section{References}

1. C. M. Oldenburg, S. E. Borglin, G. J. Moridis, Transport in Porous Media 38 (2000) 